\title{
Effect of Home Grinding on Properties of Brewed Coffee
}

\author{
Christopher Murray ${ }^{1} \&$ Thamara Laredo $^{1,2}$ \\ ${ }^{1}$ Department of Sustainability Sciences, Lakehead University, Orillia, Canada \\ ${ }^{2}$ Department of Chemistry, Lakehead University, Orillia, Canada \\ Correspondence: Christopher Murray, Department of Sustainability Sciences, Lakehead University, Orillia, ON., \\ L3V 0B9, Canada. Tel: 1-705-330-4008. E-mail: cmurray1@lakeheadu.ca
}

Received: October 5, 2014 Accepted: November 6, 2014 Online Published: November 19, 2014

doi:10.5539/jfr.v4n1p77 URL: http://dx.doi.org/10.5539/jfr.v4n1p77

\begin{abstract}
We present measurements of particle size distribution, density, loss of coffee on brewing and caffeine content in brewed coffee (as measured using Fourier Transform Infrared Spectroscopy) as a function of grinding time using a blade-type grinder. In general, there is not a lack of correlation between coffee properties and grinding for grinding times in excess of $42 \mathrm{~s}$, but mass loss on brewing and caffeine content are both increased with grinding times between 0 and $42 \mathrm{~s}$. In addition, we present evidence that this dependence of the composition of brewed coffee on grinding time is a function of increased coffee particle surface area that results from grinding, rather than increased loss of grounds into the brewed beverage or increased percolation time. Finally, we present a general recommendation for determining equivalency between small amounts of finely ground coffee and larger amounts of coarser-ground coffee.
\end{abstract}

Keywords: coffee, grinding, caffeine, brewing, spectroscopy, absorption

\section{Introduction}

\subsection{Motivation}

Grinding the coffee beans is a necessary step prior to brewing, which can be achieved in different ways: through a blade grinder, where the beans are cut into small particles by means of a high speed turning blade, which creates a particle size distribution that is usually broad and subject to variability based on somewhat arbitrary grinding time; and through burr grinding, which is generally regarded as offering better control and producing a grind of greater consistency (or a narrower distribution of ground particle sizes). Despite these advantages, a quick search in Amazon.com's Best Sellers, Coffee Grinder category, shows that 9 out of the top 20 coffee grinders are blade-type and 7 are electric burr grinders. At an average price of $\$ 22$ it's easy to see why the blade grinders are popular: the average electric burr grinder costs more than $\$ 100$ and even manual burr grinders typically cost over $\$ 40$ (Amazon, 2013).

\subsection{Previous Examinations of Coffee Properties}

Numerous studies (Heckmann et al., 2010) have been devoted to examining the quality of brewed coffee, including characterization of the influence of roasting (Budryn et al., 2009; Crozier et al., 2012; Hecimovic et al., 2011), generalized grinding techniques (Baggenstoss et al., 2008; Bell et al., 1996), and bean origin (Crozier et al., 2012; Belay et al., 2008; Fox et al., 2013; Tello et al., 2011) on the properties of the final beverage. Work has been conducted in examination of the effect of the bean particle size on the coffee properties, but only one study has shown how coffee drinkers can influence the properties of home-brewed coffee in this way (Bell et al., 1996).

The work of Bell, Wetzel and Grand (1996) examined the caffeine content of coffee brewed as a function of time of grinding, and as a function of whether the beans were ground in-store or at home. Their results indicated that while for store-ground coffee the caffeine content is not significantly influenced on the degree of grinding, when ground at home the caffeine content of the resulting coffee increases as grinding time is increased from 8 to 18 seconds.

\subsection{Experimental Approach}

In this study, Attenuated Total Reflection (ATR)-Fourier transform infrared (FTIR) spectroscopy has been used to analyze the extractable components in coffee resulting from percolation through grounds prepared with a wide 
range of well-defined grinding times. Using FTIR is not uncommon in published studies of coffee (Karoui et al., 2010; Paradkar \& Irudayaraj, 2002; Reis et al., 2013; Singh et al., 1998). Since ATR-FTIR involves no separation of the coffee components (as takes place in any chromatography-based technique), a particular region of spectra may be influenced by the presence of more than one coffee component. A correlation of caffeine with the selected spectral region has been demonstrated through comparison of spectra obtained from caffeinated and decaffeinated beans.

The particle size distribution of ground coffee samples was examined as a function of grinding time, in order to determine the extent to which particle size can be controlled and whether there is a threshold grinding time beyond which further grinding makes little difference. For each grinding time the content of extractable compounds (that are present in caffeinated beans but absent in decaffeinated ones) in the resulting brewed coffee was determined. These measurements enable a recommendation with which coffee drinkers may attempt to mimize the money they spend on coffee without sacrificing the amount of caffeine they consume. Alternatively this could be viewed as an estimation of how many cups of coffee made following the blade grinder instructions are be needed for an equivalent caffeine content prepared using longer grind times.

Although particle size distribution is measured in this study, it is reasonable to assume that the surface area of the coffee particles, rather than their absolute size, directly affects caffeine content in the brewed coffee. Though surface area was not measured, it can be approximated based upon measurements of particle size. Since the surface area of a particle is propotional to the square of the particle size and the number of particles derived from a given bean is proportional to the inverse cube of the particle size, we can expect total surface area of a ground coffee sample to be approximately proportional to the inverse of the average particle size, such that a tenfold reduction in particle size should correspond to a tenfold increase in surface area.

While the dependence of extractable compounds on grinding time is of direct importance to coffee drinkers that grind beans at home, it is also of interest to determine which of the many factors that change with grinding are responsible for changes in extractable compound concentration. As such, we have examined the dependence of the caffeine content of brewed coffee on two factors that increase with longer grinding times: percolation time and the presence of grounds in the brewed coffee. While percolation time and the amount of grounds that pass the filter into the brewed coffee are not directly influenced by grinding (they are a function of several aspects of the brewing process, including the brewing device used), it is reasonable to expect that finer particles resulting from longer grinding will contribute to longer percolation times and a higher fraction of particles fine enough to pass the filter, as well as greater surface area for a given mass of coffee. By considering the effect of increased percolation time and grounds in the brewed coffee separately, we have attempted to discern the impact of these two factors relative to particle size (and inferred surface area). As particle shape is not characterized, any change in particle surface area inferred from measured changes in particle size is approximate.

\section{Materials and Methods}

\subsection{Sample Preparation}

Melitta $^{\mathrm{TM}}$ brand (Estate Whole Bean, 100\% Arabica, medium roast) coffee beans were used for the majority of samples prepared in this study. For one control sample, decaffeinated beans (Ethical Bean, British Columbia, $100 \%$ Arabica, $99.9 \%$ decaffeinated by supercritical fluid extraction) were used. In every sample, $37 \pm 0.5 \mathrm{~g}$ of whole beans were used.

Coffee of different particle size distributions was prepared by grinding using a Black and Decker SmartGrind ${ }^{\text {TM }}$ (model CBG100SC) blade-type grinder (Figure 1a, b), modified to include a timed relay capable of reproducibly adjusting the grinding time interval. For the purposes of this study, grinding times between $1.5 \mathrm{~s}$ and 84 seconds were used. For one sample, no grinding was used. After grinding, as many coffee bean particles as possible were collected from the grinder using a fine brush. A consistent effort was made to extract all coffee particles from the grinder, and measurement of the sample mass before and after grinding was made.

\subsection{Density and Particle Size Distribution Measurement}

The mass of ground coffee in a tablespoon as a function of grinding time was measured, since most recommendations by coffee bean producers and coffee brewing equipment manufacturers are made based on volume, rather than mass (owing to the typical equipment available to homeowners and the precision with which typical kitchen scales can make measurements). Though each sample of brewed coffee was prepared using a consistent mass of coffee, it is reasonable to expect home brewers to use a consistent volume instead. As such, the relationship between bulk density and grinding time is of importance in predicting the effect of grinding on caffeine content of the resultant coffee. The average mass of a level tablespoon was measured for each sample by 
collecting no less than eight tablespoons, leveled off with a straightedge and taking the average of the resulting eight measurements.

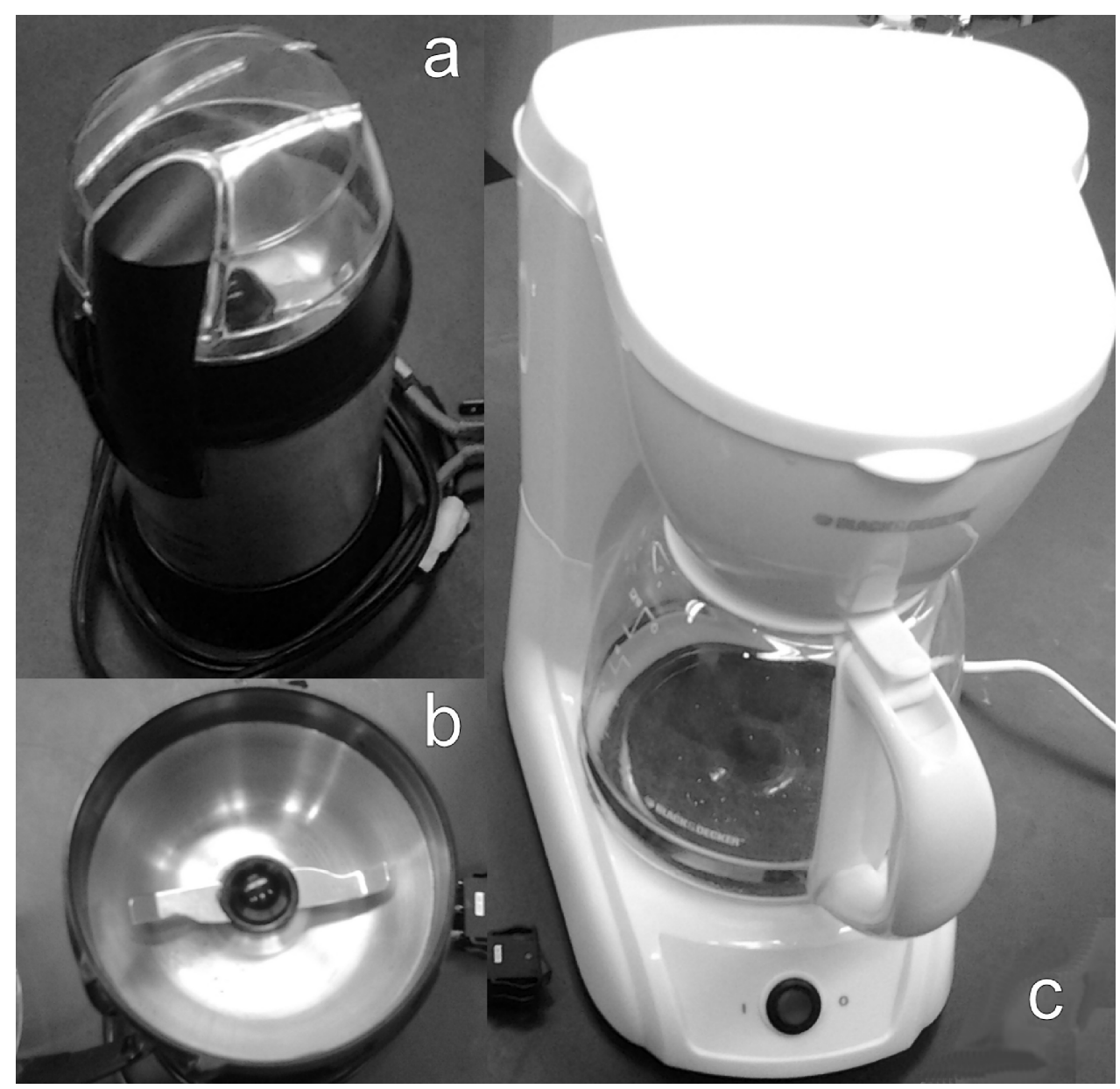

Figure 1. (a) Coffee grinder used for this study (showing (b) close up of blade) and (c) percolator-type coffee maker used

Particle size distribution for every sample was determined using a series of eight sieves, with opening sizes ranging from $9.5 \mathrm{~mm}$ to 75 microns. Each sample was separated into fractions using these sieves and the mass of every fraction was measured. The composite mass of these fractions was determined and compared with the mass of the sample before sieve analysis.

The ground coffee samples were placed in a commonly available paper coffee filter and inserted into a standard (Black and Decker, Model \#CM1200WC) coffee percolator (Figure 1c) for brewing using $20 \mathrm{ml}$ of tap water for every gram of coffee (as measured when adding to the filter, after grinding and particle size analysis). This amount of water was chosen based on the grinder manufacturer's recommendation of between 1.18 and $1.47 \mathrm{~L}$ of water for a $60 \mathrm{~g}$ sample of ground coffee. The time required for the brewing to complete was recorded and between batches a blank run of 12 cups $(1.75 \mathrm{~L})$ of tap water was run. An estimated nominal pore size of approximately 100 microns was found for the coffee filter through optical microscopy (Figure 2). The time for percolation to complete and the volume of coffee collected was measured. 


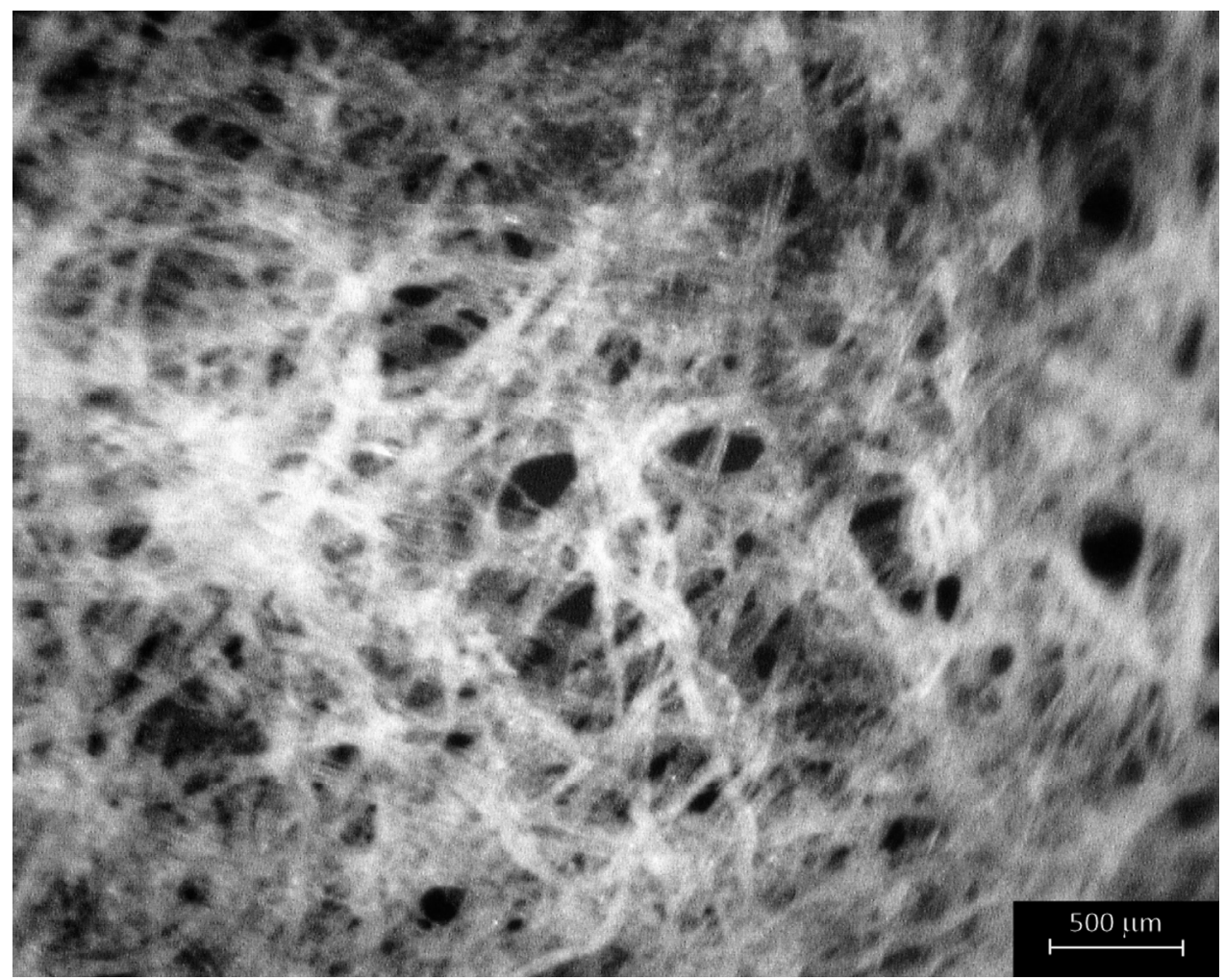

Figure 2. Optical micrograph of representative coffee filter paper used. 500 micron bar shown

A subset of three samples were measured to determine which of the several factors that change with grinding time might are most influential on the quality of the resulting coffee. Coffee beans that had been ground for five seconds were used to prepare brewed coffee as described above, but for two samples the time between brewing and extraction of the caffeine was varied considerably: for one sample, extraction took place within five minutes of brewing. For another, extraction took place more than 20 hours after the coffee was brewed. In this way, the effect of grinds that had made it past the filter and into the coffee was determined. In a final sample (which had also been ground for five seconds) the opening on the percolator was restricted so that the percolation time was increased by approximately $20 \%$, relative to the percolation time normally required for coffee with that amount of grinding.

\subsection{Extraction}

$10 \mathrm{ml}$ of chloroform were mixed with $80 \mathrm{ml}$ of brewed coffee in a $100 \mathrm{ml}$ separation funnel. Slow mixing through funnel inversions was done for three minutes. Built up pressure was released at regular intervals. The chloroform (bottom) layer was removed and allowed to sit in a covered beaker for 20 minutes. The chloroform was then fully evaporated by heating to $100{ }^{\circ} \mathrm{C}$ over a hotplate. This formed a layer of yellow crystals on the bottom of the beaker, as is shown in Figure 3. The crystals were then re-dissolved in $1 \mathrm{ml}$ of chloroform and ATR spectra were collected immediately.

\subsection{ATR Spectroscopy}

A Nexus 6700 FTIR spectrophotometer (Thermo) equipped with a DTGS detector and an ATR attachment (ATRMax, PIKE Technologies) was used to collect IR spectra at room temperature. The ATR attachment was set at a $45^{\circ}$ angle of incidence and fitted with a $45^{\circ} \mathrm{ZnSe}$ window. The resolution of all spectra was set to 4 wavenumbers. A total of 32 spectra were acquired for each sample. Pure chloroform spectra were used as a blank and to ratio against the sample in order to remove the solvent contribution. The bands between $1620-1730 \mathrm{~cm}^{-1}$ were used to monitor the chloroform extractable compounds in each sample. This region contains peaks that arise due to the $\mathrm{C}=\mathrm{O}$ stretching vibrations at 1705 and $1657 \mathrm{~cm}^{-1}$. It should be noted that some overlap with other chloroform-extractable compounds besides caffeine may also occur in this region. As is described above, one sample obtained by brewing with ground decaffeinated beans was measured and used as a control. While this method does not allow us to specify what other compounds may give rise to absorption in this region of the spectra, it does allow us to confirm the extent to which we are measuring components specific to caffeinated 
coffee. It may be that there are materials both extractable by chloroform and the super-critical solvent extraction used in the decaffeinating process that similarly give rise to absorption in the same spectral region, and our measurements would not be sensitive to the difference between these compounds. If other such extractable materials are present, they may provide sensorial properties to the final drink and as such, we have chosen to not exclude the possibility of their presence when performing the analysis. Peak areas for this region were determined using the peak area tool of the OMNIC software package (Thermo, v6.0 a). All sample spectra were collected at least in duplicate and demonstrated no more than a $\pm 5 \%$ variation in peak areas.

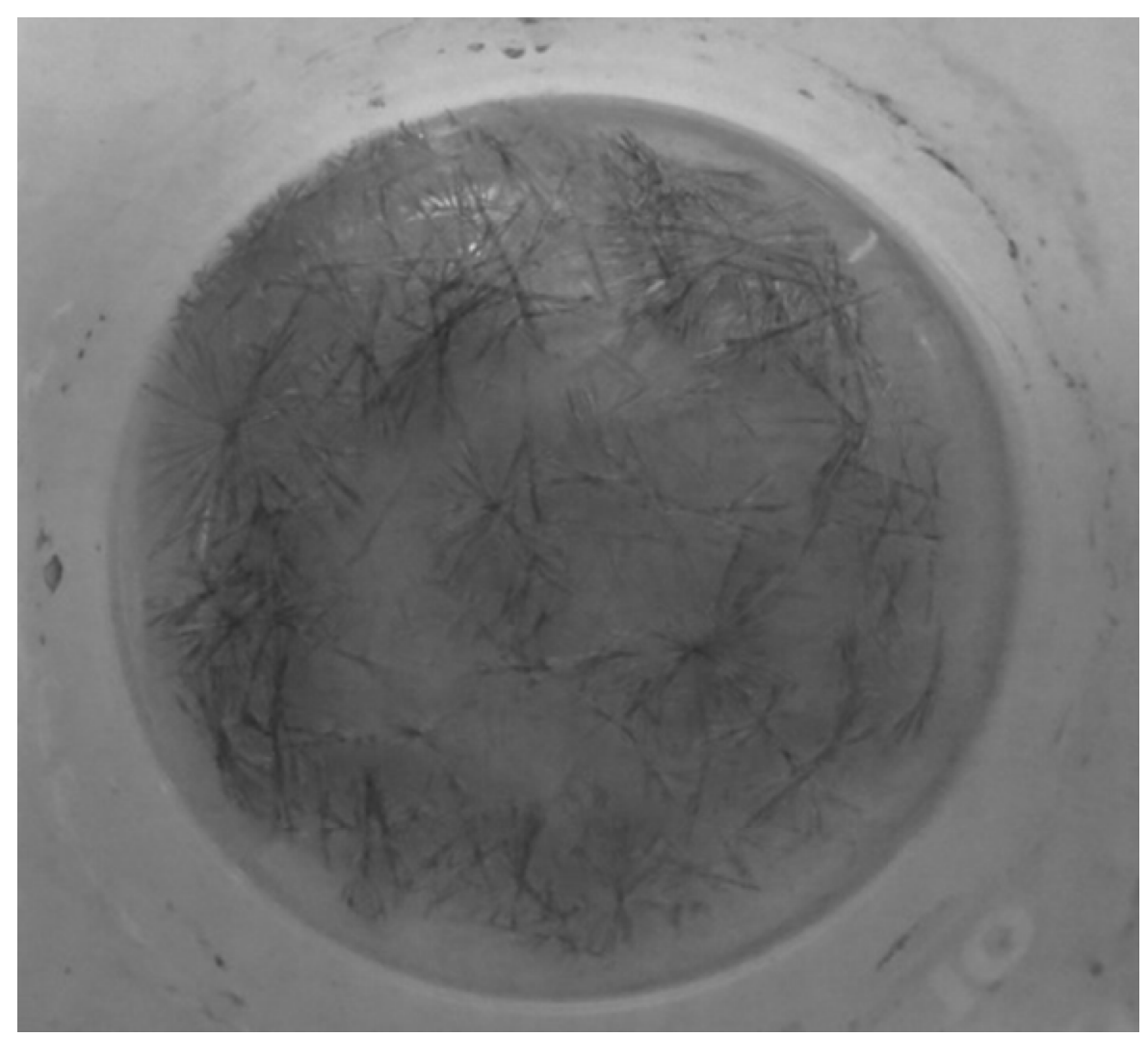

Figure 3. Sample obtained by chloroform extraction of brewed coffee and evaporation. After redissolving in choloroform samples were placed onto the ATR cell

After brewing, the coffee and filter were dried in an oven and the mass after drying was measured to determine loss of the sample upon brewing.

\section{Results and Discussion}

Loss of mass on grinding increased from 0.03 to $0.9 \%$ with increasing grinding time (Figure 4 ). Absolute values of loss one might expect will vary with the method employed to extract bean particles from the grinder, but in general a finer grind led to more sample being lost as the grinder was opened.

A small increase in density (as measured by the average mass in a Tablespoon measurement, shown in Figure 5 was observed with increased grinding time, but this increase was only slightly larger than the standard deviation amongst repeated measurements of the same sample.

As is reported elsewhere (Bell et al., 1996), the particle size distribution we measured through sieve analysis shifted to smaller values with increased grinding time (Figure 6). The distribution also became more narrow and asymmetric with increasing grinding times, implying a lower limit to the particle size achievable with this method of grinding (Figure 6). While initial (unground) bean samples had an average size between 6 and $8 \mathrm{~mm}$, after 84 seconds of grinding, the majority of bean particles were of size between 75 and 300 microns (with $4 \%$ smaller than 75 microns). 


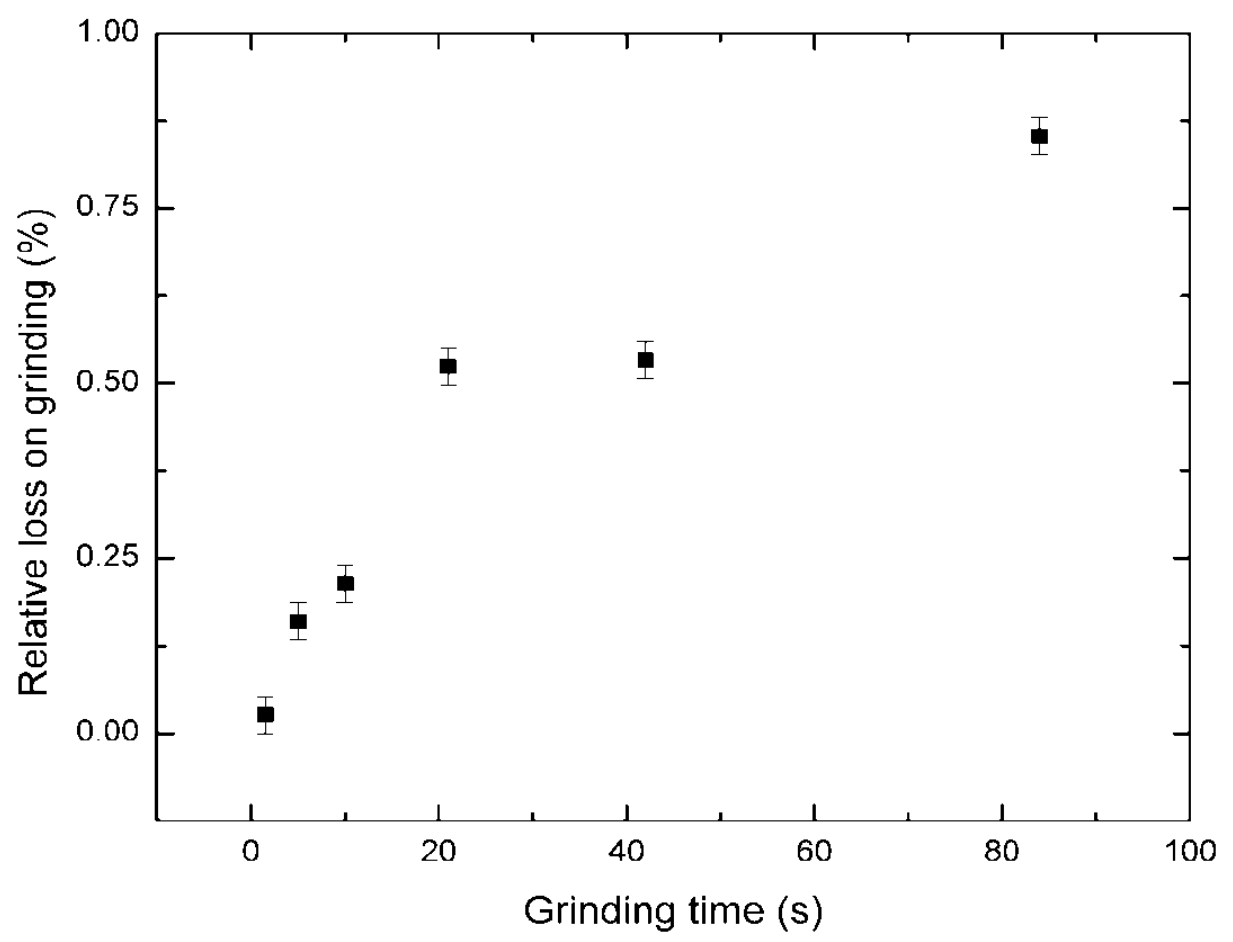

Figure 4. Relative loss of sample mass measured upon grinding, as a function of grinding time

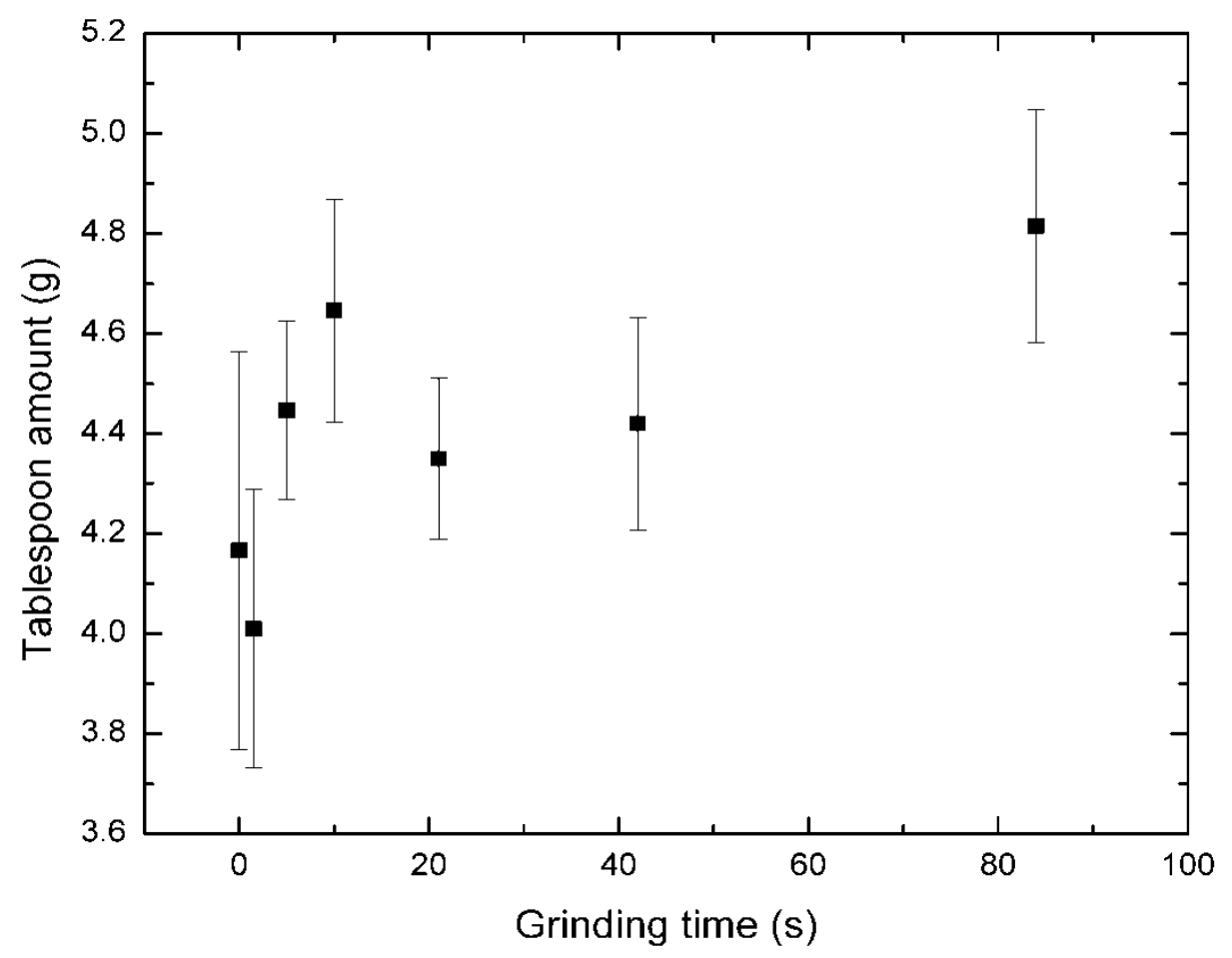

Figure 5. Average mass of ground coffee in a level tablespoon (error bars indicate standard deviation in at least eight duplicate measurements) as a function of grinding time 


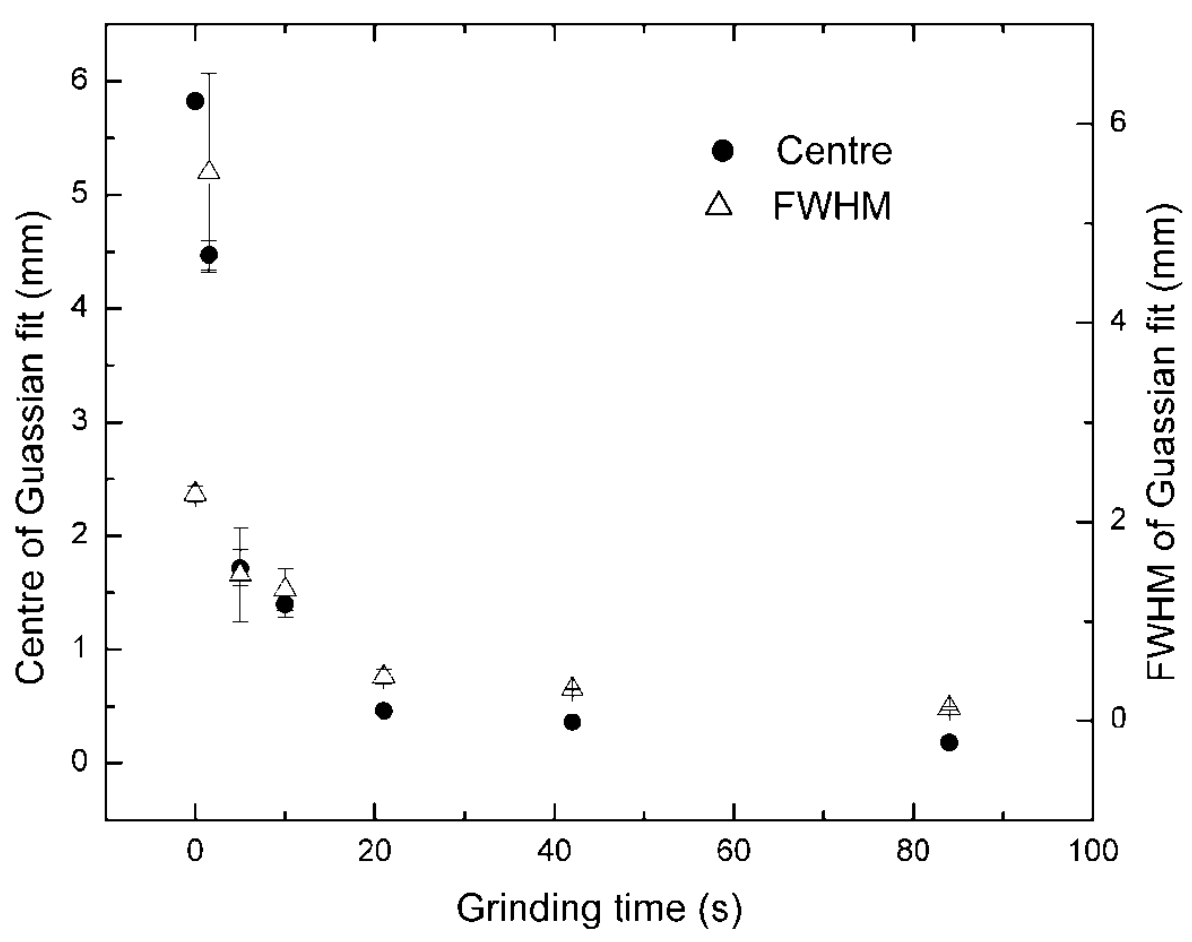

Figure 6. Centre of Guassian peak fit to the particle size distributions (left) and FWHM of those fits (right), as a function of grinding time

A representative FTIR-ATR spectra indicating the region used to determine the presence of caffeine is shown in Figure 7. A positive correlation between the amount of caffeine and grinding time was measured up until 42 seconds of grinding (Figure 8), and for 84 seconds of grinding no correlation between caffeine and grinding time was observed. After grinding for 42 seconds (which is a long time when compared with manufacturer recommendation of 10 seconds for a full $60 \mathrm{~g}$ sample but likely to occur with some statistical significance amongst home brewers) the extractable content was almost 3.5 times higher than that measured with 1.5 seconds of grinding, and more than double that obtained after 5 seconds of grinding (a typical time for a small sample mass of $37.5 \mathrm{~g}$ ). Both unground beans and a control sample of decaffeinated coffee yielded values of extractable content indistinguishable from zero. These results suggest that while there is an upper limit to the effect increased grinding times may have on the qualities of the brewed coffee (above 42 seconds, in this study), the change in the properties of coffee that can be obtained by grinding beyond the manufacturers' recommendations are greater than has previously been noted.

A positive correlation between the amount of coffee lost during the brewing process and grinding time was measured (Figure 9). Even for unground coffee, $4 \%$ of the dry mass was lost during the brewing process, and with increased grinding this value increased to more than $27 \%$. The result is consistent with the measured coffee filter pore size of approximately 100 microns (Figure 2) and suggests that further grinding past 42 seconds is not likely to significantly increase this loss upon brewing.

A weak increase in the time required for all the water to percolate through the grounds was observed with increased grinding time (Figure 10). The volume of water lost upon brewing (or conversely, the volume of water retained by the grounds after percolation) was observed to increase as the grinding time increased (Figure 11), suggesting that the finer ground coffee provided more contact area with which to interact with water, allowing more water to be contained in the saturated grounds. 


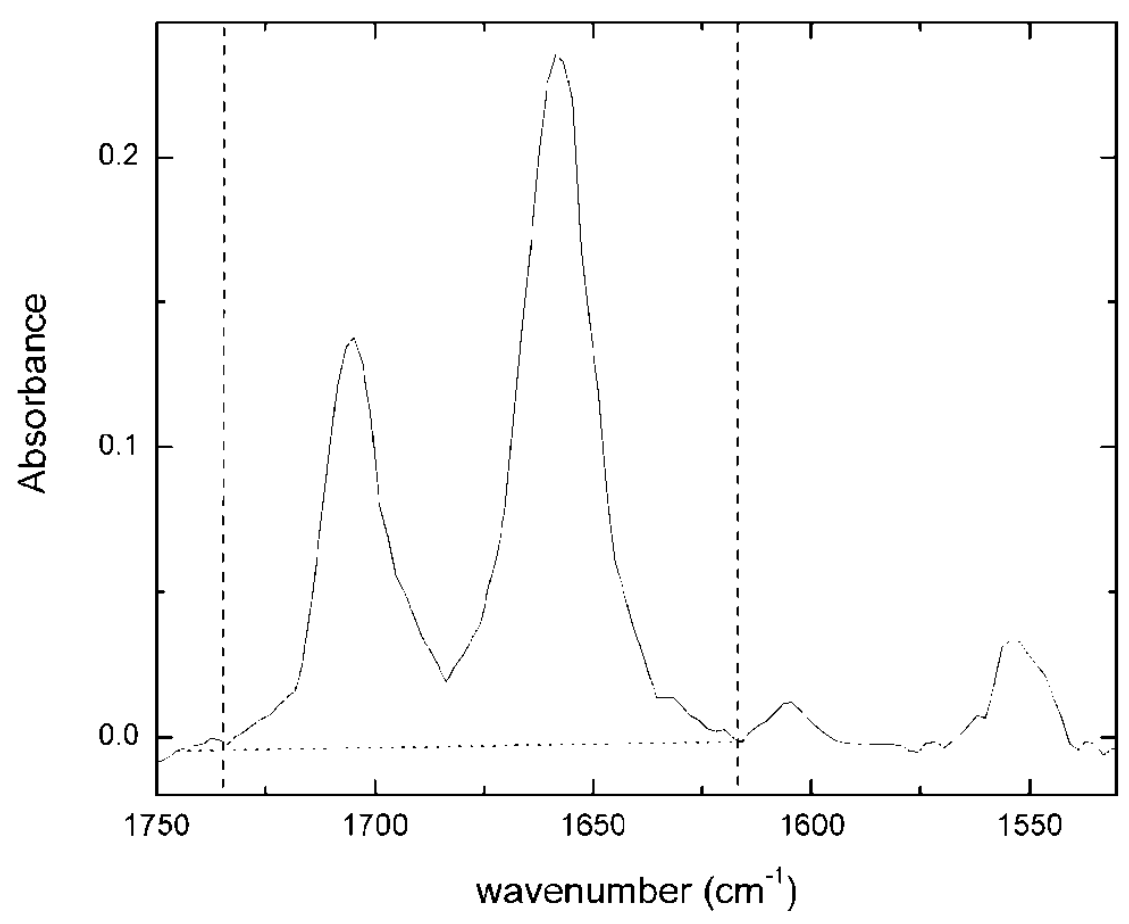

Figure 7. Region of absorbance spectra used for determination of caffeine content. Vertical dashed lines indicate the peak area (between 1735.6 and $1618 \mathrm{~cm}^{-1}$ ) used for the determination, and dotted line indicates the baseline (from 1744.2 to $1616.2 \mathrm{~cm}^{-1}$ ) subtracted for all measurements. Data shown corresponds to extract from coffee prepared from beans with $10 \mathrm{~s}$ of grinding

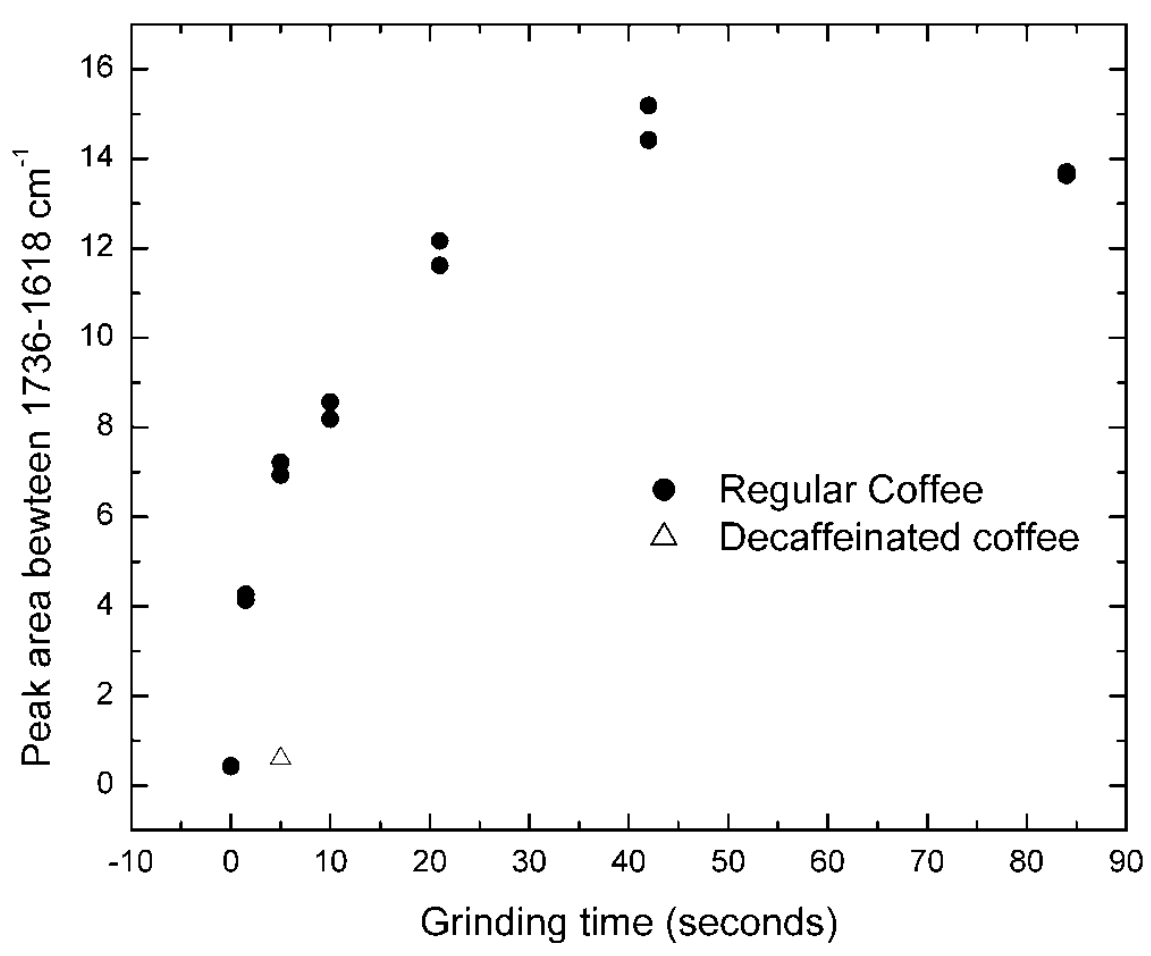

Figure 8. The area of peaks in the 1735.6 to $1618 \mathrm{~cm}^{-1}$ region of infrared absorption spectra as shown in Figure 6 . Measurement corresponding to coffee brewed using decaffeinated beans (ground for 5 seconds) is shown as the open triangle point 


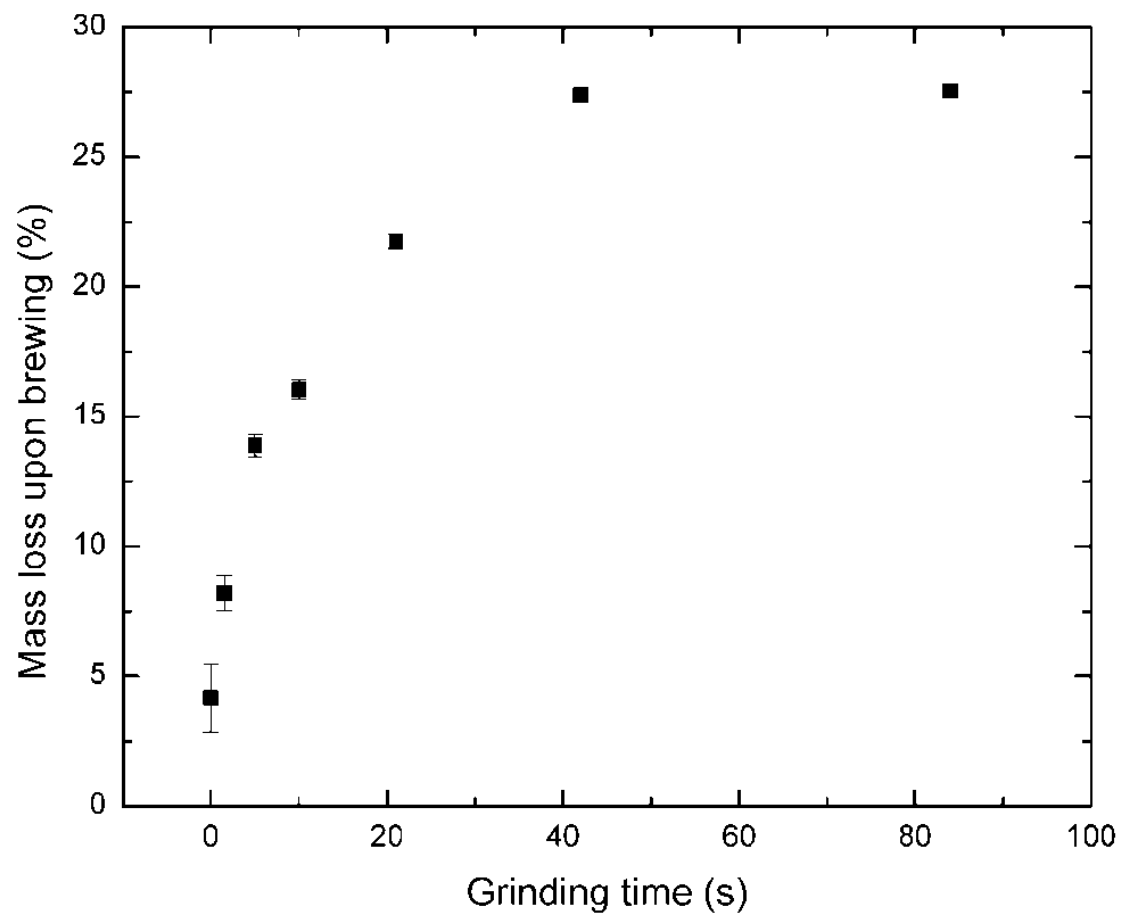

Figure 9. Relative mass loss upon brewing, as a function of grinding time. Relative mass loss values were calculated by dividing the difference between dried ground coffee beans and filter before and after brewing

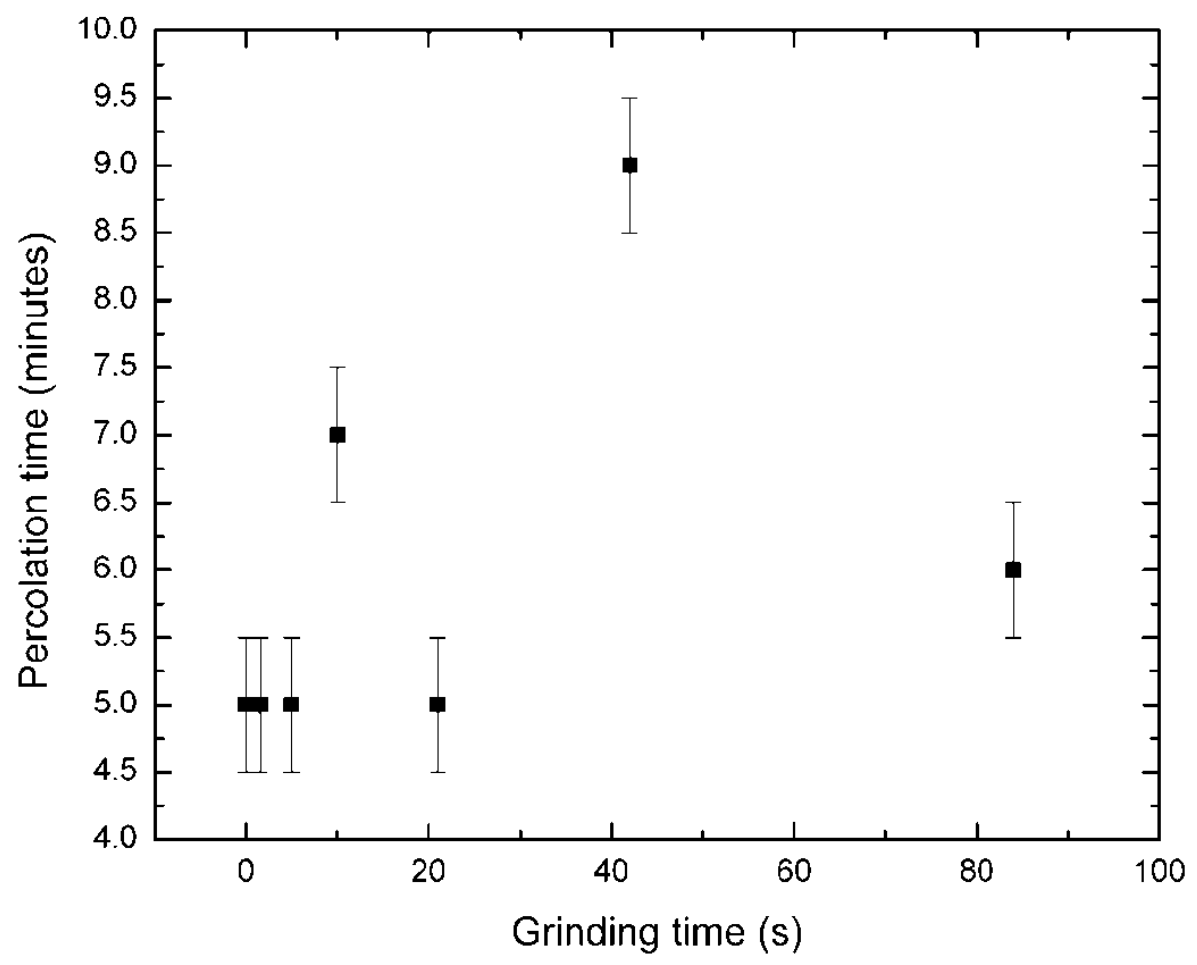

Figure 10. Time required for complete batch of coffee to percolate, as a function of grinding time for the beans used to prepare the coffee 


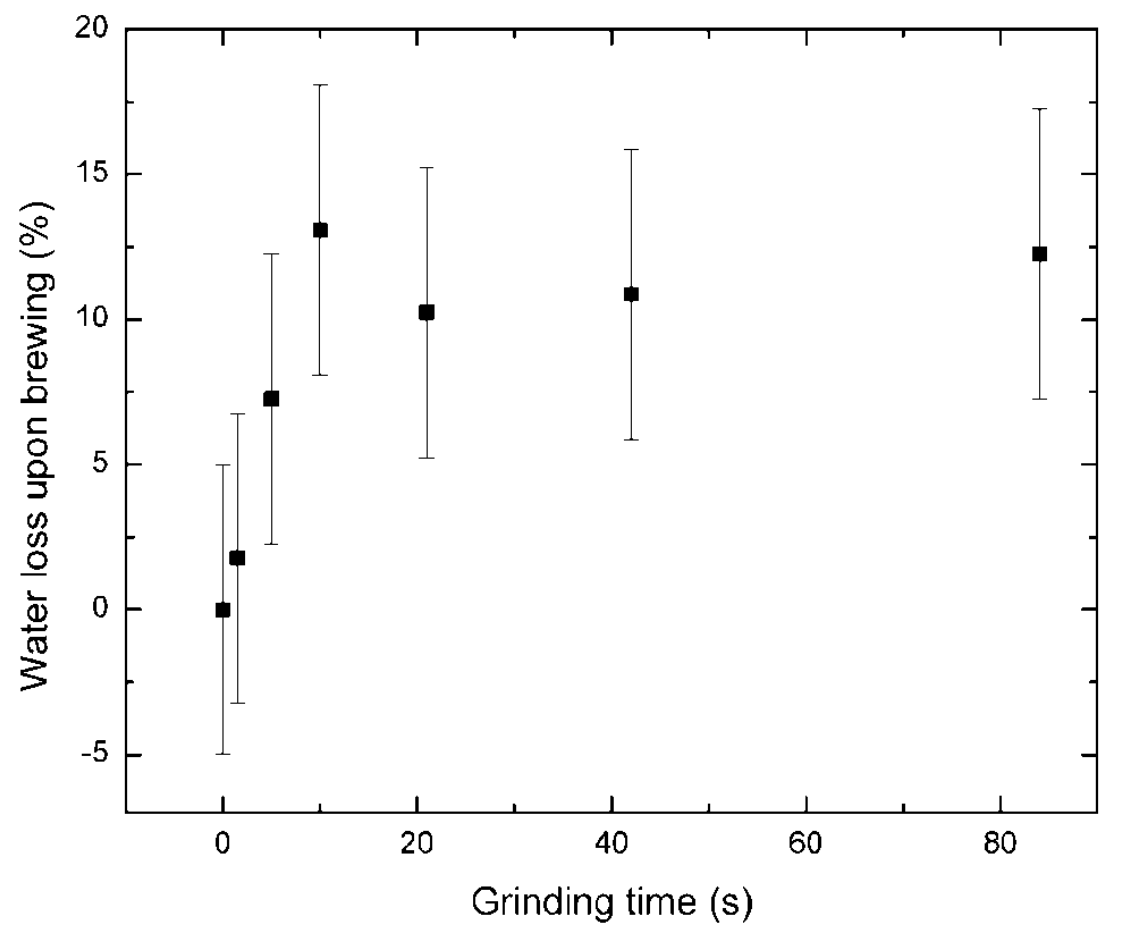

Figure 11. Water loss measured in brewing (or conversely, the percentage of water retained in the grinds after percolation was complete) as a function of bean grinding time

Two samples of coffee ground for 5 seconds each were used to determine the effect of grounds that pass the filter and into the brewed coffee. For one sample, the solvent extraction was performed less than five minutes after brewing was complete. In another otherwise identical sample, the brewed coffee was allowed to sit for more than 20 hours before solvent extraction was performed. There was no difference between the extractable compound content in the two samples (to within the resolution of the experiment).

A final sample of beans was ground for five seconds and brewed following the above procedure using a modification to the coffee percolator basket that restricted flow and prolonged percolation by more than $20 \%$. Solvent extraction was performed on the sample within five minutes of brewing and the extractable content was indistinguishable from that of other samples with $5 \mathrm{~s}$ of grinding and shorter percolation times.

As there are several properties of the ground coffee that vary with grinding time, it is not obvious from the results indicated in Figure 8 which factor have the greatest influence on caffeine content. Particle size (and the associated surface to volume ratio), mass loss upon brewing, water retained by the grounds after percolation and percolation time all show a positive correlation with grinding time. If increasing amounts of grounds passing the filter into the brewed coffee with longer grinding times (as is suggested to occur by the measurements shown in Figure 9) affect caffeine content, one would expect that more time for any grounds that pass the filter to remain in contact with the water before extraction would result in higher caffeine concentrations. This is not observed. If the slightly longer percolation time observed with finer particles was responsible for greater caffeine content we would expect that forcing the percolation time to be longer (while fixing the particle size distribution of the coffee beans) would lead to higher caffeine content. As this, too, was not observed, we can conclude that decreased particle size and the increased surface area available for contact with water during brewing dominates the resulting caffeine content. This is consistent with the results shown in Figure 11, indicating that the surface area available to contact (and retain) water in the finely ground samples is higher. This deeper understanding of the nature of the observed caffeine increase with grinding is of importance to further development of grinding practices, and to home brewers that have the ability to vary both the grinding time and the residence time coffee spends in contact with the grounds (as can be done using a French press, for example).

The results described above allow us to estimate how much coffee could be saved by grinding for longer times (if similar caffeine content were the primary aim). Though the dependence of caffeine content and loss with brewing on grinding time is not linear, a simple rule of thumb can be developed to estimate the effect of this 
dependence. In general, 42 seconds of grinding will provide double the caffeine content and double the mass loss upon brewing obtained with 5 seconds of grinding. A home brewer could make use of this information by either drinking smaller cups of coffee while achieving the same effect (making one pot last twice as long) or by using less coffee beans for every pot (allowing one's supply of coffee to last twice as long). It is also information of import to those required to restrict their caffeine intake, as grinding beans for too long may lead to much higher caffeine content than expected.

\section{References}

Amazon. (2013). Amazon Best Sellers: Best Coffee Grinders. Retrieved from http://www.amazon.com/gp/bestsellers/home-garden/289750/ref=pd_zg_hrsr_hg_1_4_last.

Baggenstoss, J., Perren, R., \& Escher, F. (2008). Water content of roasted coffee: impact on grinding behaviour, extraction, and aroma retention. European Food Research and Technology, 227, 1357-1365. http://dx.doi.org/10.1007/s00217-008-0852-8

Belay, A., Ture, K., Redi, M., \& Asfaw, A. (2008). Measurement of caffeine in coffee beans with UV/vis spectrometer. Food Chemistry, 108, 310-315. http://dx.doi.org/10.1016/j.foodchem.2007.10.024

Bell, L., Wetzel, C., \& Grand, A. (1996). Caffeine content in coffee as influenced by grinding and brewing $\begin{array}{llll}\text { techniques. } & \text { Food } & \text { Research } & \text { International, }\end{array}$ http://dx.doi.org/10.1016/S0963-9969(97)00002-1

Budryn, G., Nebesny, E., Podsddek, A., Ôyrelewicz, D., Materska, M., Jankowski, S., \& Janda, B. (2009). Effect of different extraction methods on the recovery of chlorogenic acids, caffeine and Maillard reaction products in coffee beans. European Food Research and Technology, 228, 913-922. http://dx.doi.org/10.1007/s00217-008-1004-x

Crozier, T., Stalmach, A., Lean, M., \& Crozier, A. (2012). Espresso coffees, caffeine and chlorogenic acid intake: potential health Implications. Food \& Function, 3, 30. http://dx.doi.org/10.1039/C1FO10240K

Fox, G., Wu, A., Liang, Y., \& Force, L. (2013). Variation in caffeine concentration in single coffee beans. Journal of Agricultural and Food Chemistry, 61, 10772-10778. http://dx.doi.org/10.1021/jf4011388

Hecimovic, I., Belscak-Cvitanovic, A., Horzic, D., \& Komes, D. (2011). Comparative study of polyphenols and caffeine in different coffee varieties affected by the degree of roasting. Food Chemistry, 129, 991-1000. http://dx.doi.org/10.1016/j.foodchem.2011.05.059

Heckman, M., Weil, J., \& Gonzalez de Mejia, E. (2010). Caffeine (1, 3, 7-trimethylxanthine) in Foods: A Comprehensive Review on Consumption, Functionality, Safety, and Regulatory Matters. Journal of Food Science, 75, 77-87. http://dx.doi.org/10.1111/j.1750-3841.2010.01561.x

Karoui, R., Downey, G., \& Blecker, G. (2010). Mid-Infrared Spectroscopy Coupled with Chemometrics: A Tool for the Analysis of Intact Food Systems and the Exploration of Their Molecular Structure-Quality Relationships - A Review. Chemical Reviews, 110, 6144-68. http://dx.doi.org/10.1021/cr100090k

Paradkar, M., \& Irudayara, J. (2002). A Rapid FTIR Spectroscopic Method for Estimation of Caffeine in Soft Drinks and Total Methylxanthines in Tea and Coffee. Journal of Food Science, 67, 2507-2511. http://dx.doi.org/10.1111/j.1365-2621.2002.tb08767.x

Reis, N., Franca, A., \& Oliviera, L. (2013). Discrimination between roasted coffee, roasted corn and coffee husks by Diffuse Reflectance Infrared Fourier Transform Spectroscopy. LWT - Food Science and Technology, 50, 715e722. http://dx.doi.org/10.1016/j.lwt.2012.07.016

Singh, B., Wechter, M., Hu, Y., \& Lafontaine, C. (1998). Determination of caffeine content in coffee using Fourier transform infra-red spectroscopy in combination with attenuated total reflectance technique: a bioanalytical chemistry experiment for Biochemists. Biochemical Education, 26, 243-247. http://dx.doi.org/10.1016/S0307-4412(98)00078-8

Tello, J., Viguera, M., \& Calvo, L. (2011). Extraction of caffeine from Robusta coffee (Coffea canephora var. Robusta) husks using supercritical carbon dioxide. Journal of Supercritical Fluids, 59, 53-60. http://dx.doi.org/10.1016/j.supflu.2011.07.018

\section{Copyrights}

Copyright for this article is retained by the author(s), with first publication rights granted to the journal.

This is an open-access article distributed under the terms and conditions of the Creative Commons Attribution license (http://creativecommons.org/licenses/by/3.0/). 